\title{
A Case of Capecitabine-Induced Sarcoidosis
}

\author{
Shin Myung Kang, M.D. ${ }^{1,2}$, Ji Yeon Baek, M.D. ${ }^{3}$, Bin Hwangbo, M.D. ${ }^{1}$, Hyae-Young Kim, M.D. ${ }^{1}$, Geon-Kook \\ Lee, M.D. ${ }^{1}$, Hee Seok Lee, M.D. ${ }^{1}$ \\ ${ }^{1}$ Center for Lung Cancer, National Cancer Center, Goyang, ${ }^{2}$ Department of Pulmonary and Critical Care Medicine, Gachon \\ University Gil Hospital, Incheon, ${ }^{3}$ Center for Colorectal Cancer, National Cancer Center, Goyang, Korea
}

\begin{abstract}
Sarcoidosis is an inflammatory disease involving multiple-organs with an unknown cause. The new onset of sarcoidosis associated with therapeutic agents has been observed in 3 clinical settings; tumor necrosis factor antagonists in autoimmune rheumatologic diseases, interferon alpha with or without ribavirin in patients with chronic hepatitis C or melanoma, and antineoplastic agent-associated sarcoidosis in patients with hematologic malignancies. Here, we report a female patient who developed sarcoidosis after capecitabine treatment as an adjuvant chemotherapy for sigmoid colon cancer. To our knowledge, this is the first report of a capecitabineinduced sarcoidosis.
\end{abstract}

Key Words: Sarcoidosis; Capecitabine; Sigmoid Neoplasms

\section{Introduction}

Sarcoidosis is a systemic granulomatous inflammatory disease of unknown etiology ${ }^{1,2}$. A Case Control Etiologic Study of Sarcoidosis identified positive association of sarcoidosis with agricultural employment, insecticides exposure, and mold/mildew exposure at work ${ }^{3,4}$. Genetic and immunologic factors also have been reported to be associated with sarcoidosis ${ }^{1,2}$. Capecitabine is an oral prodrug of 5-fluorouracil. Clinical efficacy of capecitabine has been demonstrated in colorectal cancer and safety profiles of capecitabine also well-established $^{5}$. Common toxic effects of capecitabine are diarrhea, nausea, vomiting, stomatitis, and hand-foot syndrome ${ }^{5}$.

Here, we report a female patient who developed sarcoidosis after capecitabine treatment as adjuvant chemotherapy for sigmoid colon cancer. To our knowledge,

Address for correspondence: Hee Seok Lee, M.D. Center for Lung Cancer, National Cancer Center, 323, Ilsan-ro, Ilsandong-gu, Goyang 410-769, Korea

Phone: 82-31-920-1749, Fax: 82-31-920-1520

E-mail: jekyde7@gmail.com

Received: Jan. 12, 2012

Revised: Jan. 20, 2012

Accepted: Jan. 27, 2012 this is the first report of a capecitabine-induced sarcoidosis.

\section{Case Report}

A 50-year-old female patient was referred to pulmonary specialist for further evaluation of the chest lesions observed in chest X-ray and chest computed tomography (CT). Seven months ago, she was diagnosed with sigmoid colon cancer. The patient underwent complete surgical resection of sigmoid colon cancer (pT3NOMO, stage IIA) followed by eight cycles of adjuvant chemotherapy with capecitabine (Xeloda ${ }^{\circledR}$; Roche, Basel, Switzerland; $1,250 \mathrm{mg} / \mathrm{m}^{2}$ twice daily for 4 cycles, thereafter $937 \mathrm{mg} / \mathrm{m}^{2}$ twice daily). Before the diagnosis of sigmoid colon cancer, she was a healthy woman and a life-time never-smoker. She had no respiratory symptoms. The physical examination over whole body was noncontributory.

Chest radiography showed bilateral hilar adenopathies and parenchymal infiltrates in left upper lung field (Figure 1B). Chest CT scan exhibited multiple lymph nodes enlargement: right supraclavicular, right upper paratracheal, prevascular, right and left lower paratracheal, subaortic, paraaortic, subcarinal, right and 

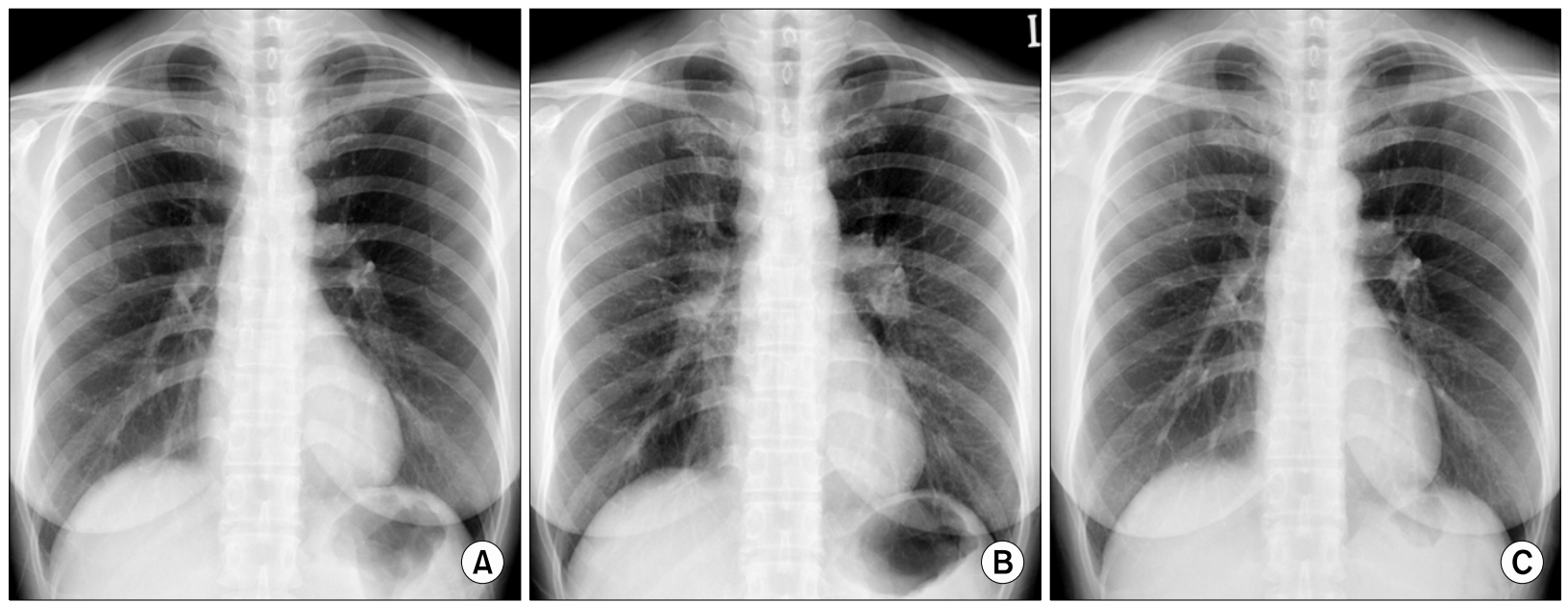

Figure 1. Chest radiography prior to capecitabine chemotherapy (A) shows no abnormality. After chemotherapy (B), bilateral hilar adenopathies and parenchymal infiltrates in the left upper lung field are observed. Three months after the discontinuation of capecitabine (C), bilateral hilar adenopathies and parenchymal infiltrates are not observed.
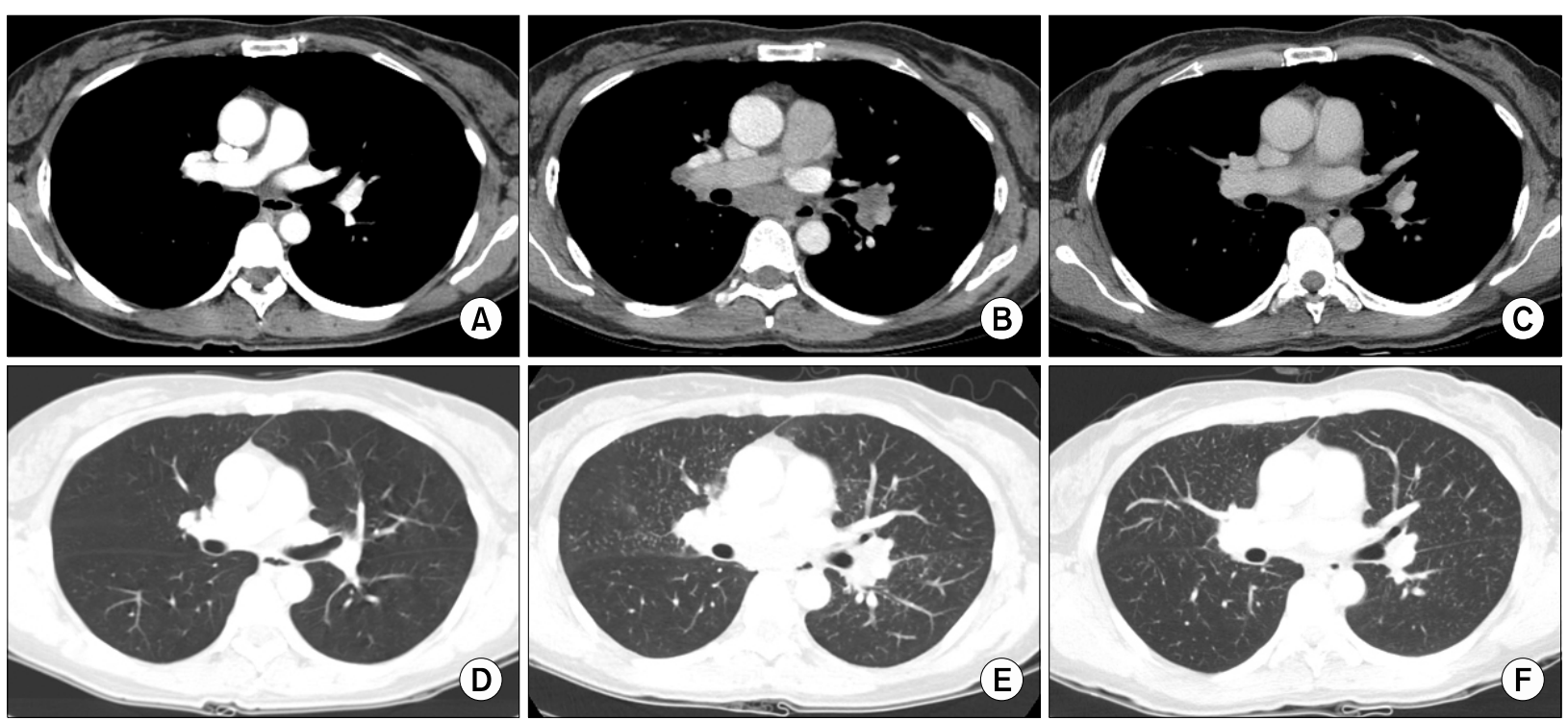

Figure 2. There are no unusual findings in the lungs and mediastinum regarding the chest computed tomography (CT) taken before capecitabine chemotherapy (A, D). After the chemotherapy (B, E), subcarinal and hilar lymph nodes enlargement, beaded bronchovascular bundle, and multiple scattered tiny nodules are observed on the chest $\mathrm{CT}$. Three months after the discontinuation of capecitabine (C, F), the CT shows no subcarinal or hilar lymph node enlargement, and a decrease in size and the number of tiny nodules compared with previous CT images.

left hilar, and right and left interlobar lymph node. Scattered tiny nodules along with perilymphatic distribution and interlobular septal thickenings were also observed on chest CT scan (Figure 2B). However, there were no abnormal findings on chest radiography and CT scan at initial diagnosis of sigmoid colon cancer
(Figures 1A, 2A). A positron emission tomography (PET)-CT scan displayed enlarged hypermetabolic lymphadenopathies in right supraclavicular, mediastinal, hilar, and retroperitoneal areas (Figure 3B). PET-CT scan performed initially showed no abnormal hypermetabolic lesion except in sigmoid colon (Figure 3A). 
Laboratory findings showed white blood cell count of $5,280 / \mu \mathrm{L}$, calcium of $9.6 \mathrm{mg} / \mathrm{dL}$ (reference range, 8.8 $\sim 10.5 \mathrm{mg} / \mathrm{dL}$ ), serum angiotensin converting enzyme level of $38.8 \mathrm{U} / \mathrm{L}$ (reference range, $20 \sim 70 \mathrm{U} / \mathrm{L}$ ). Tests for hepatitis $B$ virus surface antigen or hepatitis $C$ virus antibody were negative. Serum carcinoembryonic antigen level of $1.1 \mathrm{ng} / \mathrm{mL}$ was the same to the baseline level at diagnosis (reference range, $0 \sim 5.0 \mathrm{ng} / \mathrm{mL}$ ).

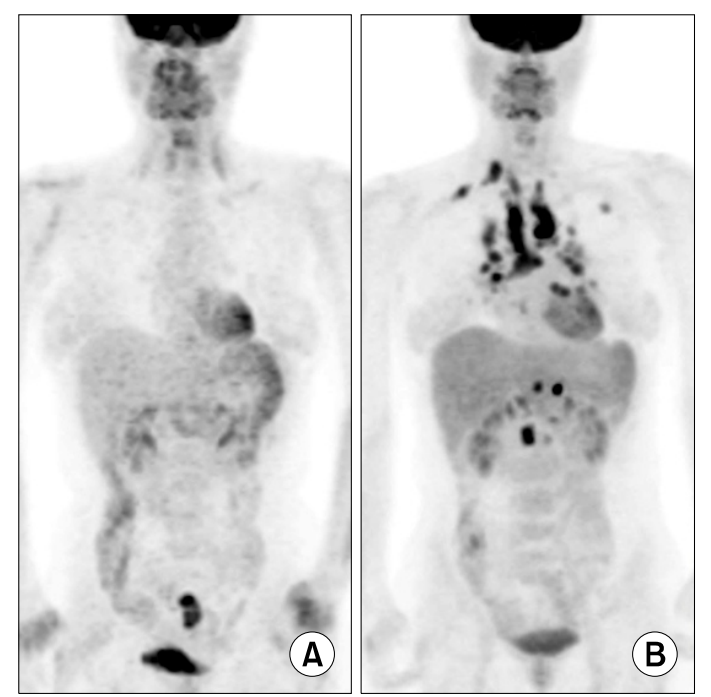

Figure 3. There is no abnormal hypermetabolic lesion except in the sigmoid colon before capecitabine chemotherapy on positiron emission tomography-computed tomography scan (A). After the chemotherapy (B), multiple hypermetabolic lesions suggesting lymphadenopathies are observed in right supraclavicular, mediastinal, hilar, and retroperitoneal areas. A hypermetabolic lesion is also observed in the left upper lung field.
Pulmonary function test and diffusion capacity of the lung for carbon monoxide (DLco) showed normal range of values: forced vital capacity (FVC) of 2.97 L (94\% of predicted value), forced expiratory volume in the first second $\left(\mathrm{FEV}_{1}\right)$ of $2.82 \mathrm{~L}$ (118\% of predicted value), $\mathrm{FEV}_{1} / \mathrm{FVC}$ of $95 \%$, total lung capacity of $5.19 \mathrm{~L}(111 \%$ of predicted value), and DLco of $16.5 \mathrm{~mL} / \mathrm{mm} \mathrm{Hg} / \mathrm{min}$ (90\% of predicted value).

Since we thought these findings were more suggestive of granulomatous lung diseases such as sarcoidosis or tuberculosis rather than cancer relapse in the thorax, we performed bronchoscopy with bronchoalveolar lavage (BAL) and endobronchial unltrasound-transbronchial needle aspiration (EBUS-TBNA). There is no endobronchial lesion on bronchoscopy. In analysis of BAL fluid recovered from right middle lobe, predominant lymphocytosis (92.8\% of white blood cells) and increased CD4 to CD8 ratio (4.1) were observed. The pathologic assessment of airway, lung parenchyma, and mediastinal lymph node specimens obtained by normal mucosal biopsy, transbronchial lung biopsy, and EBUSTBNA revealed chronic noncaseating granulomatous inflammation in all tissue specimens (Figure 4). No malignant cells were observed in BAL cytological analysis. BAL cultures for viruses, bacteria and fungi were negative. No microorganisms were observed in the specimens by periodic acid-Schiff stain. Tuberculosis-polymerase chain reaction (PCR) and nontuberculous mycobacterial-PCR using tissue samples were negative. Pathologic findings were compatible with sarcoidosis.
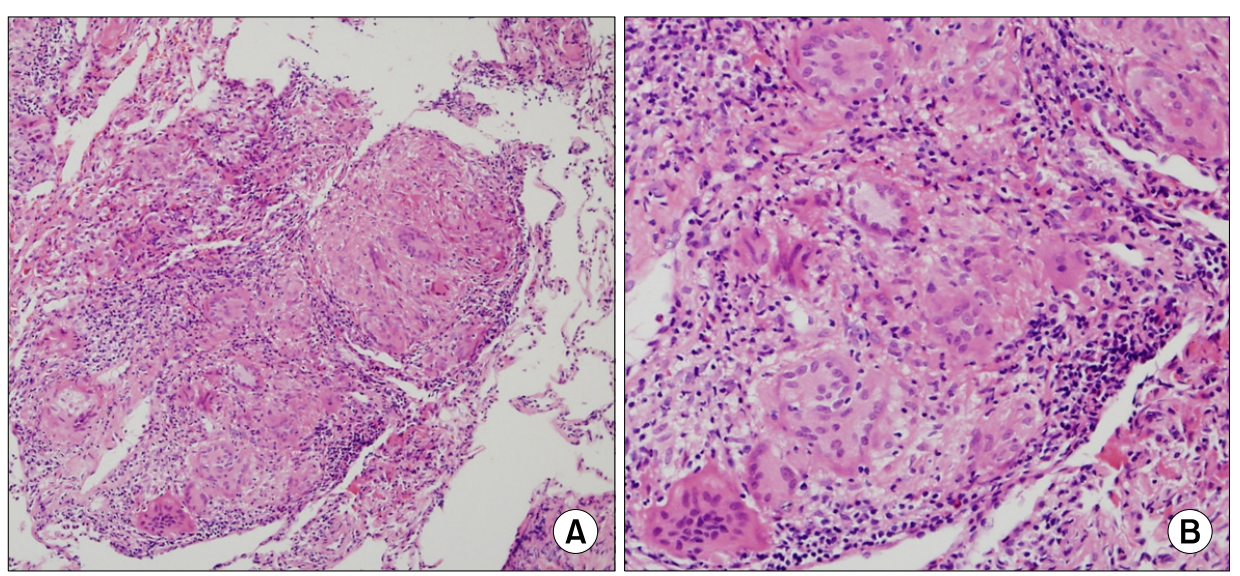

Figure 4. Microscopic finding of transbronchial lung biopsy specimens show multiple noncaseating granulomas (H\&E stain; $A$, $\times 100$; $B, \times 200$ ). 
She was asymptomatic and had stage 2 pulmonary involvement without evidence of extrapulmonary sarcoidosis except retroperitoneal lymph nodes. Moreover, she completed eight cycles of adjuvant chemotherapy with capecitabine initially planned. Therefore, we planned to observe the patient regularly without any treatment. We thought this plan might help to reveal the association between capecitabine use and new onset of sarcoidosis in this patient. Three months after the discontinuation of capecitabine, chest radiography displayed complete resolution of bilateral hilar adenopathies and parenchymal infiltrates previously noted (Figure 1C). Chest CT scan showed no subcarinal and hilar lymph nodes enlargement and decrease in size and the number of tiny nodules compared with previous CT images (Figure 2C). Finally, we concluded this case was a capecitabine-induced sarcoidosis.

\section{Discussion}

Sarcoidosis is a systemic granulomatous inflammatory disease. The cause of sarcoidosis remains unknown. Moldy environment, exposure to insecticides, Mycobacterium tuberculosis catalase-peroxidase (mKatG) protein, single-nucleotide polymorphism (rs2076530 G $\rightarrow$ A) of butyrophillin-like 2 (BTLN2) gene, annexin A11 gene on chromosome 10q22.3, some of DR subtype of class 2 human leukocyte antigens, reduced number of natural killer $\mathrm{T}$ cells have been reported to be associated with sarcoidosis $^{1-4}$. However, sarcoidosis remains a diagnosis of exclusion supported mainly by a tissue specimen demonstrating noncaseating granulomas.

The new onset of sarcoidosis associated with therapeutic agents has been observed in patient in specific 3 clinical settings. The first setting is related with the use of tumor necrosis factor (TNF) antagonists in autoimmune rheumatologic diseases, ${ }^{6,7}$. Since TNF is an important cytokine in granuloma formation in sarcoidosis, TNF-antagonist is expected to have therapeutic potentials for sarcoidosis. Paradoxically, TNF-antagonists, including infliximab and etanercept, induced sarcoidosis or sarcoid-like diseases in autoimmune rheumatologic diseases $^{6,7}$. The second setting is related with interferon alpha with or without ribavirin in patient with chronic hepatitis $\mathrm{C}$ or melanoma ${ }^{8.11}$. The third setting is antineoplastic agent-associated sarcoidosis in patient with hematologic malignancies, such as lymphoma and leukemia, or solid tumors ${ }^{12,13}$. In addition to combination chemotherapy for lymphomas, single agent of cisplatin also contributed development of sarcoidosis in cancer patients $^{13,14}$. However, there is no report on the development of sarcoidosis associated with capecitabine use up to now.

Capecitabine was highly suspected for the cause of development of sarcoidosis in this case based on clinical courses displayed newly development of sarcoidosis after capecitabine use and dramatic spontaneous resolution of sarcoidosis after the discontinuation of capecitabine. Moreover, she had no symptoms and signs suggesting sarcoidosis prior to capecitabine chemotherapy. And she was not on any medications during the period of adjuvant chemotherapy except capecitabine. A drug lymphocyte stimulation test might be used to support the relation between drugs and lung diseases, but usefulness of drug lymphocyte stimulation test for capecitabine is not known. To our knowledge, this is the first report of a capecitabine-induced sarcoidosis. In the web site (www.ehealthme.com), we could find 7 cases of sarcoidosis associated with capecitabine use. However, detailed information on diagnostic evaluations including clinical, pathologic, and radiologic characteristics are unavailable in those cases.

This patient was 50-year-old female. Of note, all cases reported in the web site above mentioned were also female aged of six decades. Usually sarcoidosis occurs commonly in the third to fourth decades of life with no gender difference ${ }^{4}$. In Korea, the peak age was in the thirties but females were predominant $(64.6 \%)^{15}$. It may be noteworthy point to check whether capecitabine-associated sarcoidosis would also affect female aged of six decades specifically in the future case. This case showed multi-compartment involvement in the lung. In addition to the involvement of parenchyma and mediastinal/hilar lymph nodes, airway involvement was 
confirmed pathologically with bronchoscopic biopsy of normal-appearing airway mucosa in this case. This case had no extrapulmonary organ involvement except retroperitoneal lymph nodes.

Here, we report a female patient who newly developed sarcoidosis after capecitabine treatment as an adjuvant chemotherapy for sigmoid colon cancer and describe her clinical manifestations. Newly development of sarcoidosis should be considered in patients on capecitabine, especially in female aged of six decades.

\section{References}

1. Baughman RP, Culver DA, Judson MA. A concise review of pulmonary sarcoidosis. Am J Respir Crit Care Med 2011;183:573-81.

2. Morgenthau AS, Iannuzzi MC. Recent advances in sarcoidosis. Chest 2011;139:174-82.

3. Newman LS, Rose CS, Bresnitz EA, Rossman MD, Barnard J, Frederick M, et al. A case control etiologic study of sarcoidosis: environmental and occupational risk factors. Am J Respir Crit Care Med 2004;170: 1324-30.

4. Baughman RP, Teirstein AS, Judson MA, Rossman MD, Yeager H Jr, Bresnitz EA, et al. Clinical characteristics of patients in a case control study of sarcoidosis. Am J Respir Crit Care Med 2001;164(10 Pt 1):1885-9.

5. Mikhail SE, Sun JF, Marshall JL. Safety of capecitabine: a review. Expert Opin Drug Saf 2010;9:831-41.

6. Clementine RR, Lyman J, Zakem J, Mallepalli J, Lindsey S, Quinet R. Tumor necrosis factor-alpha antagonist-induced sarcoidosis. J Clin Rheumatol 2010;16:274-9.

7. Ramos-Casals M, Perez-Alvarez R, Perez-de-Lis M,
Xaubet A, Bosch X; BIOGEAS Study Group. Pulmonary disorders induced by monoclonal antibodies in patients with rheumatologic autoimmune diseases. Am J Med 2011;124:386-94.

8. Ramos-Casals M, Mañá J, Nardi N, Brito-Zerón P, Xaubet A, Sánchez-Tapias JM, et al. Sarcoidosis in patients with chronic hepatitis $\mathrm{C}$ virus infection: analysis of 68 cases. Medicine (Baltimore) 2005;84:69-80.

9. Gayet AR, Plaisance P, Bergmann JF, Mouly S. Development of sarcoidosis following completion of treatment for hepatitis $\mathrm{C}$ with pegylated interferon-\{alpha\}2a and ribavirin: a case report and literature review. Clin Med Res 2010;8:163-7.

10. Heinzerling LM, Anliker MD, Müller J, Schlaeppi M, von Moos R. Sarcoidosis induced by interferon-alpha in melanoma patients: incidence, clinical manifestations, and management strategies. J Immunother 2010;33: 834-9.

11. López V, Molina I, Monteagudo C, Jordá E. Cutaneous sarcoidosis developing after treatment with pegylated interferon and ribavirin: a new case and review of the literature. Int J Dermatol 2011;50:287-91.

12. Papanikolaou IC, Sharma OP. The relationship between sarcoidosis and lymphoma. Eur Respir J 2010;36: 1207-9.

13. Cohen PR, Kurzrock R. Sarcoidosis and malignancy. Clin Dermatol 2007;25:326-33.

14. Yao M, Funk GF, Goldstein DP, DeYoung BR, Graham MM. Benign lesions in cancer patients: Case 1. Sarcoidosis after chemoradiation for head and neck cancer. J Clin Oncol 2005;23:640-1.

15. Kim DS. Sarcoidosis in Korea: report of the Second Nationwide Survey. Sarcoidosis Vasc Diffuse Lung Dis 2001;18:176-80. 